\title{
UNE ESPÈCE ET UNE VARIÉTÉ NOUVELLES D'ACANTHOCÉPHALES PARASITES DES POISSONS DE MER DES CôTES DU SÉNÉGAL, ET REDESCRIPTION DE SERRASENTIS SOCIALIS (Leidy 1851) van Cleave 1924

\author{
Par Ýves J. GoLvaN
}

Nous avons étudié la collection d'Acanthocéphales parasites des Poissons de mer des côtes du Sénégal qui nous a été confiée par M. Jean Cadenat, Directeur de la Section de Biologie Marine de l'Institut Français d'Afrique Noire (1).

Cette collection comprenait les Acanthocéphales récoltés lors de l'autopsie de 73 Poissons, appartenant à 38 espèces différentes. Tous ces Poissons ont été capturés par la Section de Biologie Marine de l'I.F.A.N., de 1951 à 1954, principalement dans la région de Dakar-Gorée.

Nous donnons ici la liste de ces Poissons et des Acanthocéphales qu'ils hébergeaient (tableau I). Les résultats détaillés de cette étude seront publiés dans le Bulletin de l'I.F.A.N. Nous nous bornerons, dans la présente note, à donner les descriptions complètes d'une espèce et d'une variété nouvelles que nous avons identifiées. Nous pensons qu'il est également utile de donner une description complète d'une espèce anciennement décrite et très commune, Serrasentis socialis.

\section{Remarques générales}

1. Manque de variété de la faune des Acanthocéphales de Poissons dans cette partie de l'Atlantique

Sur 38 hôtes différents, presque tous nouveaux, nous n'avons trouvé que 6 espèces différentes d'Acanthocéphales. Ces parasites

(1) Nous prions M. J. Cadenat de bien vouloir trouver ici l'expression de nos remerciements.

Ann. de Parasitologie, T. XXXI, $\mathrm{N}^{\circ} 3 .-1956$.

15. 
se montrent, de plus, fort peu spécifiques quant au choix de leur hôte. Ils parasitent des Poissons appartenant à des familles très éloignées zoologiquement les unes des autres.

\section{$2^{\circ}$ Variations morphologiques de Rhadinorhynchus pristis}

Ces variations portent sur le nombre de files longitudinales de crochets, le nombre de crochets par file, l'existence d'un ou deux champs d'épines cuticulaires sur le corps. Mais deux caractères sont particulièrement variables : la taille et la coloration.

A) Variations de taille. - Elles peuvent aller du simple au double selon les hôtes. Chez Sardinella aurita et chez Trachinotus goreensis, le parasite est toujours de très grande taille, certaines femelles dépassant $85 \mathrm{~mm}$. de long. Chez Scomber colias et chez Dentex filosus, par contre, la longueur n'excède guère $40 \mathrm{~mm}$. Ces différences sont constantes chez un hôte donné, quels que soient la date et le lieu de la capture, la taille du Poisson et le nombre des parasites qu'il hébergeait.

B) Variations de coloration. - Chez Sardinella aurita et chez Trachinotus goreensis, ce parasite est de couleur rouge-orangé. Chez d'autres hôtes, la teinte est plus pâle ; chez d'autres, enfin, le ver est blanchâtre.

La coloration des téguments des Acanthocéphales est normalement blanchâtre, mais elle peut être modifiée par les aliments qu'ils absorbent (Meyer, 1933).

A ce propos, nous avons eu récemment un exemple particulièrement démonstratif. A l'autopsie de plusieurs Truites (Salmo trutta L.), pêchées dans un affluent de l'Aulne, à Callac (Côtes-duNord), nous avons récolté de nombreux spécimens d'Echinorhynchus clavula Dujardin 1845 de coloration blanc rosé. Le contenu intestinal des Truites ne comprenait que des Ephémères et des Vairons (Phoxinus phoxinus L.).

Nous avons retrouvé ce même parasite chez deux Truites provenant de Milly-sur-Thérain (Oise). Sa coloration était orange. Le tube digestif des Poissons ne contenait que des Gammares (Gammarus pulex), qui, sous l'action des sucs digestifs, avaient pris une couleur orangée. Il est certain que la couleur d'E. clavula était liée à l'absorption à travers sa cuticule du pigment du Crustacé. La couleur particulière de certains spécimens de Rhadinorhynchus pristis doit être due à un phénomène analogue et dépendre, en définitive, des préférences alimentaires de l'hôte. En aucun cas, la colo- 
ration d'un Acanthocéphale ne peut être un caractère utilisable dans la systématique de ce groupe, bien que quelques auteurs aient cru pouvoir y attacher quelque importance.

$3^{\circ}$ Extension de laire de répartition géographique de certaines espèces. - Cette remarque vaut pour la variété d'Illiosentis furcatus que nous décrivons ici et également pour les deux espèces du genre Acanthocephaloides que nous signalons.

Le genre Acanthocephaloides était, selon Meyer (1933), uniquement représenté en Méditerranée et en Mer Noire. R.-Ph. Dollfus (1951) a montré que ce genre se rencontrait également dans l'Océan Indien. La découverte d'A. propinquus et d'A. chabanaudi sur la côte Ouest de l'Afrique permet d'accroître considérablement le domaine de ce genre qui apparait, d'ores et déjà, comme cosmopolite.

Cependant, sa présence dans le Pacifique reste encore à démontrer, puisque les espèces japonaises décrites par Yamaguti (19351939) et Fukui et Morisita (1936) ne peuvent être considérées comme des représentants de ce genre. Cable et Quick (1954) ont d'ailleurs créé le genre Neoacanthocephaloides qui permet de les classer.

\section{ILLIOSENTIS FURCATUS var. AFRICANA n. var.}

Créé en 1939 pour Illiosentis furcatus Van Cleave et Lincicome 1939, le genre Illiosentis ne compte que deux espèces découvertes, toutes les deux, chez des Poissons d'Amérique du Nord : Illiosentis furcatus chez Menticirrhus americanus (Louisiane); Illiosentis centratus chez Menticirrhus undulatus, Roncador stearsi et Umbina roncador (Californie).

Nous avons trouvé $I$. furcatus dans l'intestin d'Albula vulpes (Albulidaæ). Ce Poisson est très commun sur les côtes du Sénégal et il semble qu'il soit très souvent parasité par 1 . furcatus, car nos exemplaires ont été récoltés chez des $A$. vulpes capturés en des points très éloignés du littoral et à des périodes différentes de l'année.

Ce parasite est très aisément identifiable, car les six crochets qui terminent les six files ventrales du proboscis sont énormes, beaucoup plus développés que tous les autres.

Dans l'espèce type, ces grands crochets mesurent de 0,051 à $0,077 \mathrm{~mm}$. de long chez les mâles et de 0,051 à $0,089 \mathrm{~mm}$. chez les femelles. De plus, les crochets de l'avant-dernière rangée circulaire 


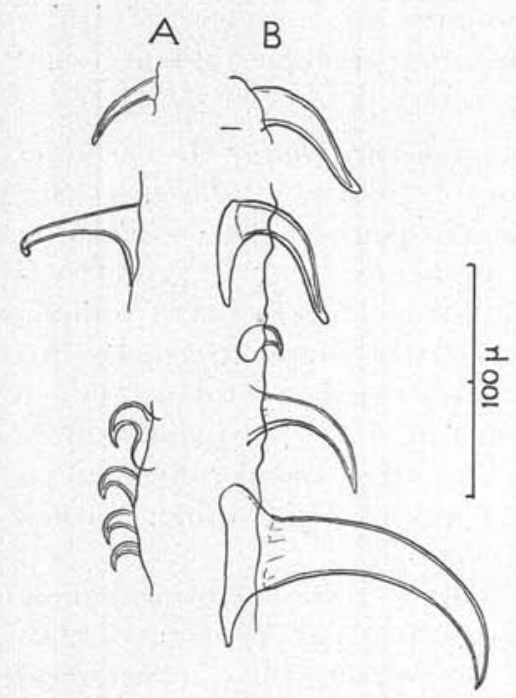

FIG. 1. - Illiosentis furcatus var. africana: Crochets du proboscis. A : file dorsale; $B$ : file ventrale. sont pratiquement de la même taille que ceux du reste du proboscis (planche I, fig. B).

Chez nos exemplaires africains, les six grands crochets basaux sont plus grands. Ils mesurent en moyenne $0,124 \mathrm{~mm}$. de long, cette dimension étant mesurée selon la technique préconisée par Van Cleave (1939). De plus, les crochets de l'avantdernière rangée circulaire sont, bien que plus petits que ceux de la base, encore nettement plus développpés que ceux du reste du proboscis (fig. 1).

La comparaison des deux schémas (planche I, fig. A et B) rend aisément compte de ces différences.

Il ne nous paraît pas souhaitable, sur ces simples détails, de créer une nouvelle espèce. En effet, aucun autre élément ne diffère suffisamment de l'espèce type pour légitimer cette création. Si nous n'avons pu voir les épines qui entourent l'orifice vulvaire, ceci est très probablement dû à l'extrême fragilité de ces ornements (Van Cleave et Lincicome, 1939).

Nous proposons pour cette variété africaine, peu différente de la variété néarctique, le nom d'Illiosentis furcatus var. africana $\mathrm{n}$. var.

\section{GORGORHYNCHUS ROBERTDOLLFUSI n. sp.}

Hôte : Gempylus serpens (Gempylidx) capturé au large de Port-Etienne (Mauritanie) en décembre 1953.

Habitat : Intestin grêle.

Matériel. - Trois femelles immatures, dont une seule espèce possède un proboscis bien évaginé. Type conservé dans la collection d'helminthologie de l'Institut de Parasitologie de la Faculté de Médecine de Paris. 


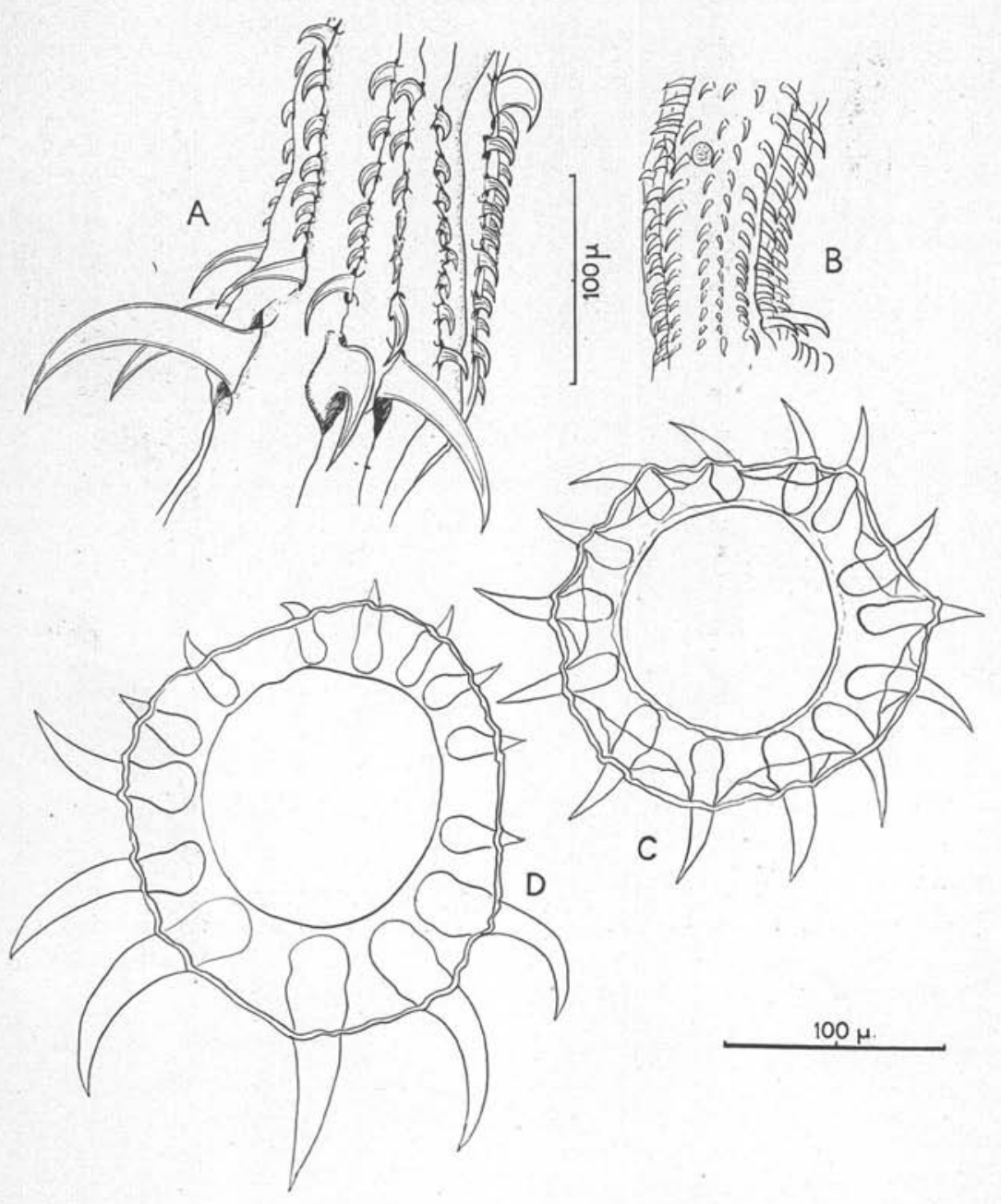

PLanche I. - Illiosenstis furcatus.

A : Partie inférieure du proboscis d'une femelle de la variété africaine ; B : Même partie du proboscis des exemplaires américains (d'après Van Cleave, 1941) ; C : Coupe transversale de la partie médiane du proboscis ; D : Coupe transversale du proboscis au niveau de la dernière rangée de crochets.

Description. - Proboscis (planche II, fig. B) : Ovalaire, à grosse extrémité postérieure, mesurant $0,6 \mathrm{~mm}$. de long sur $0,42 \mathrm{~mm}$. dans sa plus grande largeur. 


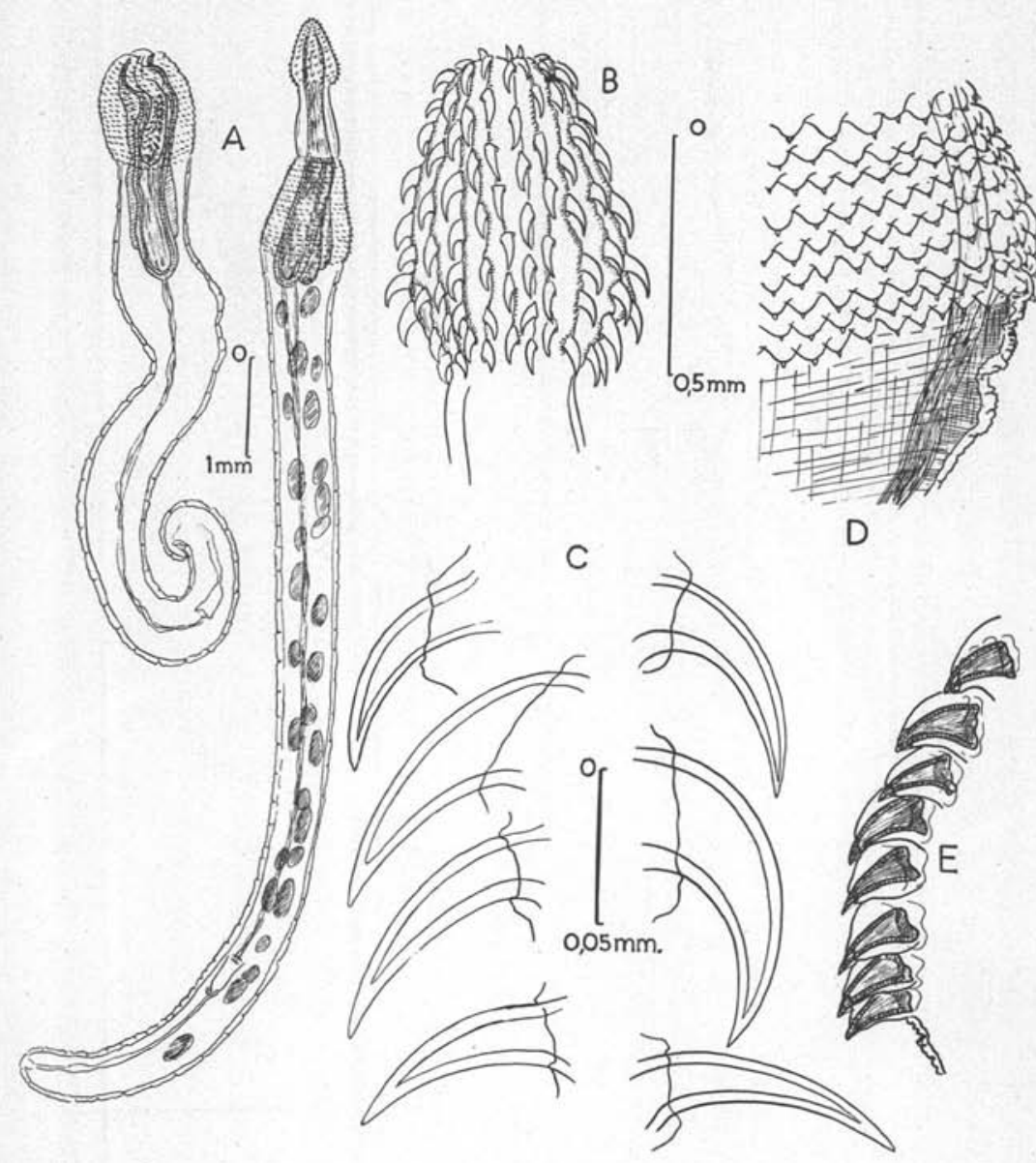

PLANCHE II. - Gorgorhynchus robertdollfusi n. sp.

A : Deux exemplaires femelles immatures ; B : Proboscis parfaitement évaginé ; C. Quelques crochets du proboscis ; D : Champ d'épines cuticulaires du corps ; E : Vue latérale des épines cuticulaires du corps.

Armé de 18 files longitudinales de 10 à 12 crochets. Nette dissymétrie entre les crochets des faces dorsale et ventrale, les crochets les plus grands étant ceux de la face ventrale, en particulier ceux de la partie moyenne élargie du proboscis. 
Dimensions en millimètres des principaux crochets du Proboscis

\begin{tabular}{|c|c|c|c|c|}
\hline \multirow[t]{2}{*}{ Rangée } & \multicolumn{2}{|c|}{ Face ventrale } & \multicolumn{2}{|c|}{ Face dorsale } \\
\hline & Longueur & Largeur & Longueur & Largeur \\
\hline & 一 & 一 & 一 & - \\
\hline I & 0,095 & 0,045 & 0,065 & 0,020 \\
\hline IV & 0,097 & 0,041 & 0,073 & 0,042 \\
\hline VII & 0,076 & 0,018 & 0,070 & 0,018 \\
\hline $\mathrm{N}$ & 0,070 & 0,015 & 0,043 & 0,015 \\
\hline
\end{tabular}

Cou: De forme tronconique régulière, il mesure $0,8 \mathrm{~mm}$. de long sur $0,26 \mathrm{~mm}$. à son point de jonction avec le proboscis et $0,38 \mathrm{~mm}$. à sa base.

Réceptacle du proboscis : Il mesure $2,10 \mathrm{~mm}$. de long sur $0,4 \mathrm{~mm}$. de large et possède une double paroi.

Ganglion cérébroïde à la partie médiane.

Lemnisci un peu plus courts que le réceptacle $(1,60 \mathrm{~mm}$. sur $0,2 \mathrm{~mm}$.).

Corps: En fuseau, dont la largeur la plus grande se trouve au niveau du $1 / 5$ antérieur et des $4 / 5$ postérieurs. La longueur maxima est de $12 \mathrm{~mm}$. La plus grande largeur est de $0,9 \mathrm{~mm}$. et elle se réduit à $0,4 \mathrm{~mm}$. dans la partie postérieure.

Un champ unique d'épines cuticulaires couvre le 1/5 antérieur du corps. Il y a de 18 à 24 rangées circulaires d'épines serrées les unes contre les autres. Chaque épine mesure en moyenne 0,022 mm. de long sur $0,013 \mathrm{~mm}$. de large à sa base. L'épine soulève un petit repli cuticulaire qui l'enveloppe à l'exception de sa pointe (planche II, fig. D et E).

Le champ d'épines cuticulaires est plus étendu sur la face ventrale $(1 \mathrm{~mm}$. de haut) que sur la face dorsale $(0,7 \mathrm{~mm}$. de haut).

Tout le reste de la surface du corps est dépourvu d'épines.

Appareil génital: N'occupe que le 1/7 postérieur du corps. La partie supérieure de l'utérus est dilatée en cloche. Cette portion est suivie de deux portions tubulaires.

Les trois exemplaires ne possédaient que des masses ovariennes sans œufs libres.

Discussion. - Cet Acanthocéphale appartient à la famille des Gorgorhynchidæ Van Cleave et Lincicome 1940 parce que :

$1^{\circ}$ Le proboscis est court, ovalaire, avec nette dissymétrie dorsoventrale des crochets. 
$2^{\circ}$ Il y a un champ unique d'épines cuticulaires au niveau de la partie antérieure du corps.

$3^{\circ}$ Le réceptacle a une double paroi avec des lemnisci sensiblement de même longueur que lui.

$4^{\circ}$ Il n'y a pas de grands crochets arqués à la base du rostre comme dans le genre Nipporhynchus Chandler 1934.

Notre espèce diffère des trois espèces actuellement décrites dans le genre Gorgorhynchus : G. gibber Chandler 1934, G. lepidus Van Cleave 1940 et G. clavatus Van Cleave 1940, par son proboscis plus court, avec un nombre de files de crochets moindre, chaque file comptant elle-même moins de crochets. De plus, les épines sont ici entourées d'un repli cuticulaire, les lemnisci sont un peu plus courts que le réceptacle.

Bien que nous n'ayons pas eu d'exemplaires mâles à notre disposition, nous considérons cependant cete espèce comme nouvelle du fait des caractères que nous venons d'énumérer et nous proposons pour elle le nom de Gorgorhynchus robertdollfusi n. sp., en hommage à notre maître, M. Robert-Ph. Dollfus.

\section{SERRASENTIS SOCIALIS (Leidy, 1851) Van Cleave, 1924 $(=$ ECHINORHYNCHUS SOCIALIS Leidy $1851=$ E. SAGITTIFER} Linton 1889)

Ce parasite est très commun partout. Il a été trouvé de l'Amérique du Nord à l'Australie (Johnston et Deland, 1929), mais il parait particulièrement abondant dans cette partie de l'Atlantique. Nous l'avons, en effet, trouvé dans la cavité générale, ou plus rarement dans l'intestin de 23 Poissons, appartenant à 12 espèces différentes.

Un fait doit être souligné, c'est que nous n'avons trouvé d'individus sexuellement mûrs que chez un seul Poisson, Elacate nigra (Rachicentridæ), capturé à Gorée, en juin 1951.

Chez tous les autres Poissons, nous n'avons observé que des adultes jeunes, mâles à testicules très petits, non fonctionnels, et femelles à ovaire divisé en masses ovariennes, mais ne contenant aucun œuf dans leur cavité pseudo-cœlomique.

La même observation a été faite par tous les auteurs qui ont étudié ce parasite et, à notre connaissance, seul Linton (1905) a vu les adultes de cette espèce et précisément chez le même hôte (Elacate nigra $=$ Rachicentron canadum). 
Van Cleave (1924) a émis l'hypothèse que $S$. socialis était un parasite qui ne se trouvait que dans certaines zones géographiquement très restreintes et que, de toute façon, il était peu abondant et presque toujours associé à une infestation massive par Echinorhynchus gadi. Cette hypothèse est, à notre avis, infirmée par le fait que nous n'avons jamais trouvé $S$. socialis associé à un autre Acanthocéphale.

La rareté des adultes n'est pas une des moindres particularités de ce curieux parasite. On doit se demander si les formes immatures deviennent ultérieurement des adultes fonctionnels ou si elles restent immatures chez tous les hôtes qui ne sont pas des Rachicentridx.

Dans le premier cas, il est difficile d'expliquer pourquoi, à aucun moment de l'année, chez tous ces hôtes, on ne trouve aucun adulte ayant terminé sa croissance et atteint son plein développement sexuel.

La seconde alternative implique une extraordinaire fécondité des femelles, puisque les juvéniles s'égarent chez plusieurs dizaines d'espèces d'hôtes d'attente ou d'hôtes accidentels.

Seule, l'étude expérimentale du cycle évolutif de cette espèce permettra de résoudre ce problème, mais l'on peut, d'ores et déjà, prédire que cette étude sera difficile à réaliser.

La description de Linton est fort incomplète. La description originale de Leidy, puis celle de Van Cleave ont trait à des juvéniles. Dans la Monographie de Meyer (1933) est figuré un juvénile mâle, mais pas d'adulte. Dans ces conditions, il nous a paru nécessaire de donner ici une description détaillée des adultes sexuellement mûrs.

Ajoutons que Ward (1954) a donné un dessin de ce parasite qu'elle a trouvé chez un nouvel hôte, Trachinotus falcatus, mais sans préciser s'il s'agissait de juvéniles ou d'adultes parfaits (1).

Matériel. - 35 individus parfaitement conservés, 24 mâles et 11 femelles, récoltés chez un seul Elacate nigra.

Description. - Espèce de grande taille; les femelles atteignent $130 \mathrm{~mm}$. de long sur $1,4 \mathrm{~mm}$. de large dans la partie antérieure et $3,5 \mathrm{~mm}$. dans la partie postérieure dilatée. Les mâles ne dépassent guère $75 \mathrm{~mm}$. de long sur $0,80 \mathrm{~mm}$. de large en avant et $1,4 \mathrm{~mm}$. de large en arrière.

(1) Une nouvelle espèce du genre Serrasentis, $S$. chauhani, a été décrite en 1954 par Datta mais nous n'avons pu consulter ce travail. 


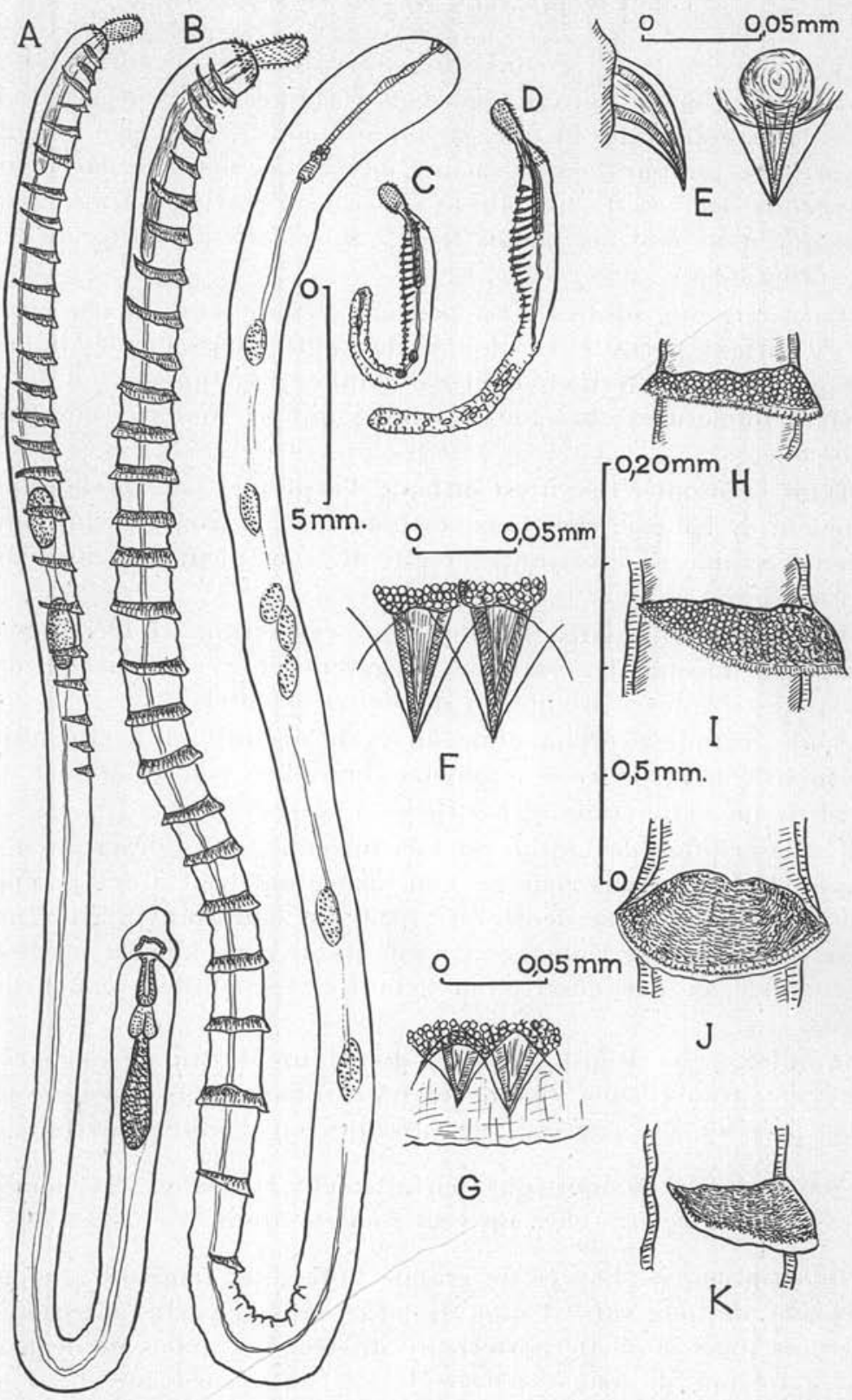

Planche III. - Serrasentis socialis.

A : Exemplaire mâle sexuellement mûr ; B : Exemplaire femelle sexuellement mûr ; C : Exemplaire màle jeune ; D : Exemplaire femelle jeune ; E : Epines cuticulaires antérieures du corps; F : Epines de la première collerette ; G : Epines de la dernière collerette ; H-I : Premières collerettes ; J-K : Dernières collerettes. 


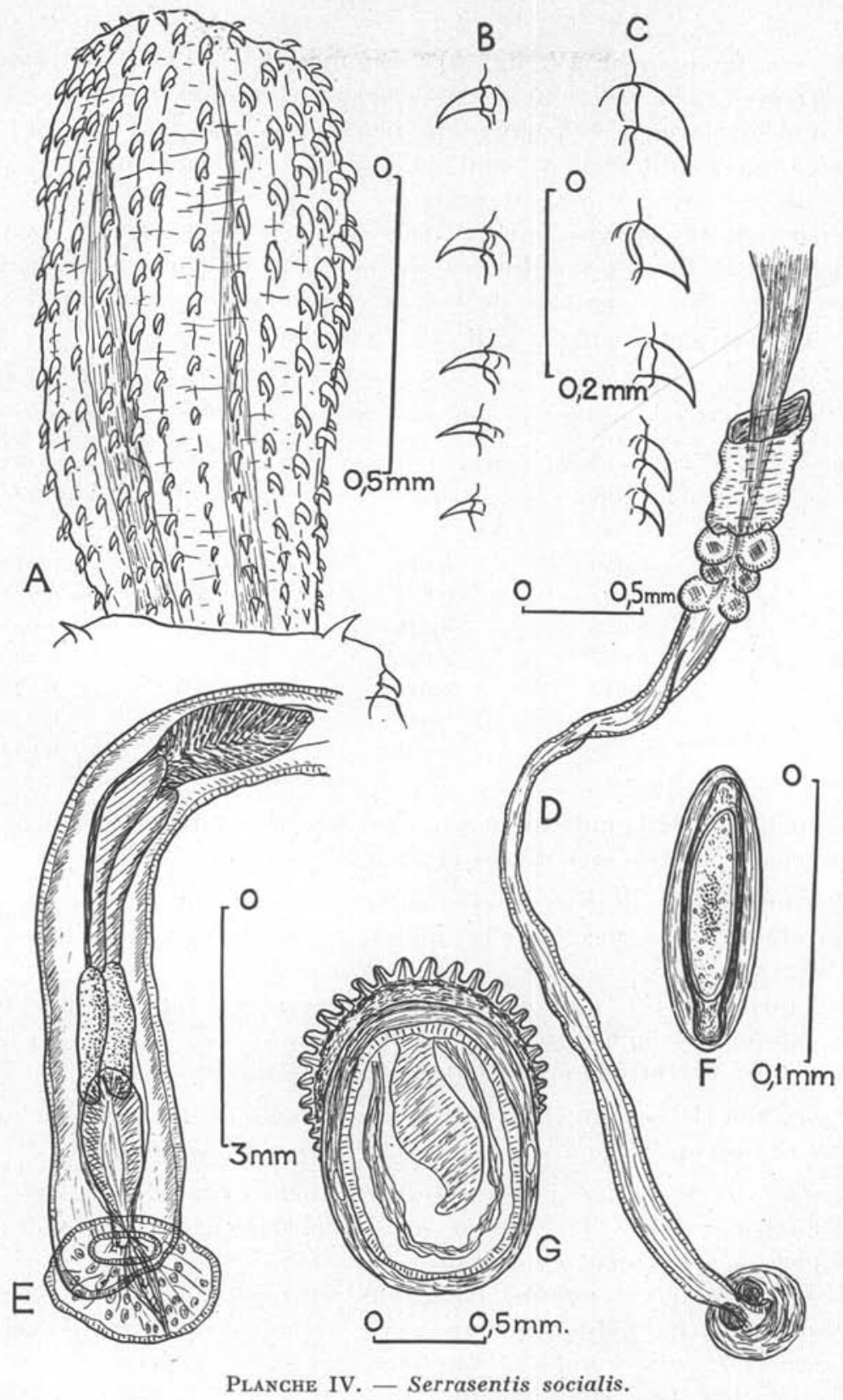

A : Proboscis d'un exemplaire femelle; B : File dorsale. Quelques crochets ; C : File ventrale. Quelques crochets ; D : Appareil utérovaginal d'une femelle adulte ; E : Appareil génital d'un mâle parfaitement adulte, avec la bourse copulatrice évaginée ; F : GEuf mûr ; G : Coupe du corps au niveau d'une des premières collerettes. 
Proboscis (planche IV, fig. A) : En massue, à grosse extrémité supérieure. Chez les femelles, il mesure $1,8 \mathrm{~mm}$. de long sur $1,2 \mathrm{~mm}$. de large à sa partie supérieure et $1 \mathrm{~mm}$. à sa base. Chez les mâles, ces dimensions sont de $1 \mathrm{~mm}$. pour la longueur sur $0,6 \mathrm{~mm}$. de large au maximum.

Proboscis fortement incliné sur la face ventrale du corps, armé de 24 files longtudinales de 14 à 18 crochets avec nette dissymétrie dorso-ventrale de ces crochets. Les crochets ventraux sont les plus développés (fig. B et C, planche IV).

Dimensions en millimètres des principaux crochets du Proboscis

\begin{tabular}{|c|c|c|c|c|}
\hline \multirow[t]{3}{*}{ Rangée } & \multicolumn{2}{|c|}{ Face ventrale } & \multicolumn{2}{|c|}{ Face dorsale } \\
\hline & Longueur & Largeur & Longueur & Largeur \\
\hline & - & 一 & 一 & - \\
\hline I & 0,050 & 0,015 & 0,040 & 0,018 \\
\hline II $\ldots$ & 0,054 & 0,021 & 0,055 & 0,028 \\
\hline IV $\ldots$ & 0,058 & 0,020 & 0,056 & 0,030 \\
\hline VIII . . & 0,047 & 0,020 & 0,058 & 0,033 \\
\hline $\mathrm{X}$ & 0,043 & 0,013 & 0,063 & 0,022 \\
\hline XIII $\ldots \ldots \ldots$ & 0,050 & 0,011 & 0,054 & 0,020 \\
\hline $\mathrm{XV} \quad \ldots \ldots \ldots$ & 0,032 & 0,009 & 0,057 & 0,014 \\
\hline
\end{tabular}

L'épaisseur de la cuticule n'a pas permis de connaître la morphologie exacte des racines de ces crochets.

Réceptacle du proboscis: Longueur $4,3 \mathrm{~mm}$. sur $0,45 \mathrm{~mm}$. de large chez les femelles. Chez les mâles, $3 \mathrm{~mm}$. de long sur $0,20 \mathrm{~mm}$. de large.

Lemnisci un peu plus courts que le réceptacle, aplatis et rubanés $(3,8 \mathrm{~mm}$. de long sur $0,25 \mathrm{~mm}$. de large).

Ganglion cérébroïde à la partie antérieure du réceptacle.

Cou : Court, de forme conique. Chez la femelle, 1,4 mm. de long sur $0,40 \mathrm{~mm}$. de large à son sommet et $0,80 \mathrm{~mm}$. de large à sa base.

Corps : Très allongé, presque régulièrement cylindrique dans ses $3 / 5$ antérieurs ; il s'élargit dans ses $2 / 5$ postérieurs pour se terminer par un renflement en massue.

Il est caractérisé, comme chez tous les représentants du genre Serrasentis, par l'existence d'une série de collerettes d'épines cuticulaires (Cuticular combs $=$ Cuticularplatten), sur environ la moitié antérieure du corps (planche III, fig. H, I, J, K).

A la partie toute antérieure du corps existe d'abord, sur une hauteur d'environ $1 \mathrm{~mm}$., un champ d'épines cuticulaires nettement distinctes les unes des autres. Ces épines sont disposées en quin- 
conce sur 7 à 12 rangées circulaires. Les plus grosses épines sont celles de la face ventrale $(0,065 \mathrm{~mm}$. de long sur $0,025 \mathrm{~mm}$. de large). Elles possèdent une racine en bulbe presque sphérique $(0,033 \mathrm{~mm}$. de large sur $0,028 \mathrm{~mm}$. de haut) (planche III, fig. E).

La première collerette cuticulaire est située à environ $0,5 \mathrm{~mm}$. au-dessous de la dernière rangée circulaire d'épines. Cette collerette est en croissant à concavité postéro-supérieure et elle embrasse les $5 / 6$ de la circonférence du corps, ne laissant libre que la partie médiane de la face dorsale du ver.

Elle est formée par un repli cuticulaire qui enveloppe complètement deux rangées d'épines accolées. Les épines de ces deux rangées s'imbriquent exactement. La collerette a, sur la face ventrale, une hauteur maxima de $0,25 \mathrm{~mm}$.

Au fur et à mesure que l'on s'éloigne de l'extrémité antérieure, la distance entre deụx collerettes augmente régulièrement $(1,8 \mathrm{~mm}$. environ entre les deux dernières collerettes). En même temps, la longueur des croissants se réduit, la dernière collerette ne couvrant guère que la face ventrale. La taille des épines qui en forment l'armature se réduit également, mais la hauteur du repli cuticulaire reste la même, si bien qu'au niveau des dernières collerettes la pointe des épines est située nettement en retrait du bord libre de ce repli.

Il y a de 26 à 28 collerettes chez les femelles et 23 chez les mâles.

Toute la moitié postérieure du corps du ver est dépourvue d'ornements cuticulaires.

Vaisseaux principaux du système lacunaire latéraux; droit un peu plus gros que le gauche. Les noyaux sous-cuticulaires sont nombreux et de petite taille.

Appareil génital mâle: Testicules placés l'un au-dessous de l'autre dans la moitié antérieure du corps, ovoïdes, mesurant $3 \mathrm{~mm}$. de long sur $1 \mathrm{~mm}$. de large. Canal déférent très long.

Le reste de l'appareil génital n'occupe que le 1/6 postérieur du corps. Quatre glandes cémentaires courtes, pyriformes, mesurant $1 \mathrm{~mm}$. de long.

Vésicule séminale pyriforme, de $2,4 \mathrm{~mm}$. de long sur $0,80 \mathrm{~mm}$. de large.

Bourse copulatrice à parois épaisses, de $1,4 \mathrm{~mm}$. de long sur $0,6 \mathrm{~mm}$. de large (planche IV, fig. E).

Appareil génital femelle (planche IV, fig. D) : Les sacs ligamentaires sont précocement rompus et la cavité pseudo-cœlomique est bourrée d'œufs à tous les stades de segmentation et de masses ovariennes en voie de fragmentation. 
CEufs à coque assez mince, mesurant $0,100 \mathrm{~mm}$. de long sur $0,031 \mathrm{~mm}$. de large (fig. E, planche IV).

L'appareil utéro-vaginal n'occupe que le $1 / 10$ postérieur du corps.

Cloche utérine ouverte en haut, mesurant $0,40 \mathrm{~mm}$. de long sur $0,20 \mathrm{~mm}$. de large.

Appareil sélecteur des œufs à grosses cellules, mesurant $0,28 \mathrm{~mm}$. de long.

Tube utérin : $1,30 \mathrm{~mm}$. de long sur $0,060 \mathrm{~mm}$. de large dans sa partie moyenne.

Vagin de $1,15 \mathrm{~mm}$. de long sur $0,10 \mathrm{~mm}$. de large. Sphincter vulvaire puissant, de $0,33 \mathrm{~mm}$. de diamètre.

\section{Conclusions}

La taille des exemplaires africains est plus grande que celle des spécimens décrits par Linton. Ils possèdent également plus de collerettes cuticulaires. Il convient donc d'apporter les modifications suivantes à la diagnose de l'espèce :

Corps de 130 à $70 \mathrm{~mm}$. de long chez les femelles, de 70 à $25 \mathrm{~mm}$. chez les mâles. De 18 à 28 collerettes d'épines cuticulaires.

Dans la liste des espèces de Poissons parasités par ce parasite figurent de nombreux hôtes nouveaux (tableau I).

\section{RÉsumé}

L'étude de cette importante collection permet d'apprécier l'importance relative des Acanthocéphales dans la faune parasitaire des Poissons des côtes du Sénégal. Sur 38 espèces différentes de Poissons, nous n'avons trouvé que six espèces d'Acanthocéphales, ce qui souligne le manque de variété des parasites de ce groupe chez les Poissons.

Deux espèces du genre Acanthocephaloides et une du genre Illiosentis sont signalées pour la première fois dans cette partie de l'Atlantique. Nous avons décrit une variété nouvelle, Illiosentis furcatus var. africana n. var., et une nouvelle espèce, Gorgorhynchus robertdollfusi n. sp.

Nous avons enfin cru nécessaire de redécrire les adultes de Serrasentis socialis qui ne se trouvent que chez Elacate nigra, alors que les juvéniles et les adultes immatures se rencontrent chez un très grand nombre de Poissons. 


\section{BIBLIOGRAPHIE}

Chander (A. C.). - 1934. A revision of the genus Rhadinorhynchus (Acanthocephala) with description of new genera and species. Parasitol., XXVI, (3), 352-358.

Cable (R. M.) et Quick (L. A.). - 1954. Some Acánthocephala from Puerto-Rico with the description of a new genus and three new species. Transac. American. Microsc. Soc., LXXIII, (4), 393-400.

Datra (M. N.). - 1953. On a new species of Acanthocephalan parasite from fishes of Bombay. Rec. Indian. Mus., LI, (1), 1954, 51-55.

Dollfus (R.-Ph.). - 1951. Le genre Acanthocephaloides Anton Meyer 1931 n'est pas seulement Méditerranéen et Pontique. Ann. Parasitol. Hum. Comp., XXVI, (5-6), 440-445.

Jehnston (T. H.) et Deland (E. W.). - 1929. Australian Acanthocephala, n 1. Census of recorded hosts and parasites. Transac. Roy. Soc. SouthAustralia, LIII, 146-154.

Leidy (J.). - 1851. Descriptions of new species of Entozoa. Proceed, Acad. Nat. Sci. Philadelphia, V, 155-156.

Linton (E.). - 1904. Parasites of fishes of Beaufort, North Carolina. Bull. Bur. Fisheries, XXIV, 1905, 321-428.

Meyer (A.). - 1932-33. Acanthocephala. Dr. H. G. Bronns Klassen und Ordnungen des Tierreichs, Leipzig, 1933.

Van Cleave (H. J.). - 1924. A critical study of the Acanthocephala described and identified by Joseph Leidy. Proceed. Acad. Nat. Sci. Philadelphia, LXXVI, 279-334.

1939. Analysis of hook measurements in the Acanthocephala. Vol. Jub. Prof. Sadao Yoshida, II, 331-336.

1940. The Acanthocephala collected by the Allan Hancock Pacific Expedition, 1934. Allan Hancock's Pacific Expeditions, II, (15), 501-527.

1941. Hook pattern on the Acanthocephalan proboscis. Quart. Rev. Biol., XVI, (2), 157-172.

Van Cleave (H. J.) et Lincicome (D. R.). - 1939. On a new genus and species of Rhadinorhynchida (Acanthocephala). Parasitol., XXXI, (4), 413-416.

1940. A reconsideration of the Acanthocephalan family Rhadinorhynchidæe. Jl. Parasitol., XXVI, (1), 75-81.

WARD (H. L.). - 1951. The species of Acanthocephala described since 1933. Part I. Jl. Tennessee Acad. Sc., XXVI, (4), 282-311.

1952. The species of Acanthocephala described since 1933. Part. II. Ibid., XXVII, (2), 131-149.

1954. Parasites of Marine fishes of the Miami region. Bull. Marine Sci. Gulf. Carribbean, IV, (3), 244-261.

Institut de Parasitologie de la Faculté de Médecine de Paris et Section de Biologie Marine de l'Institut Français d'Afrique Noire à Gorée (Sénégal). 


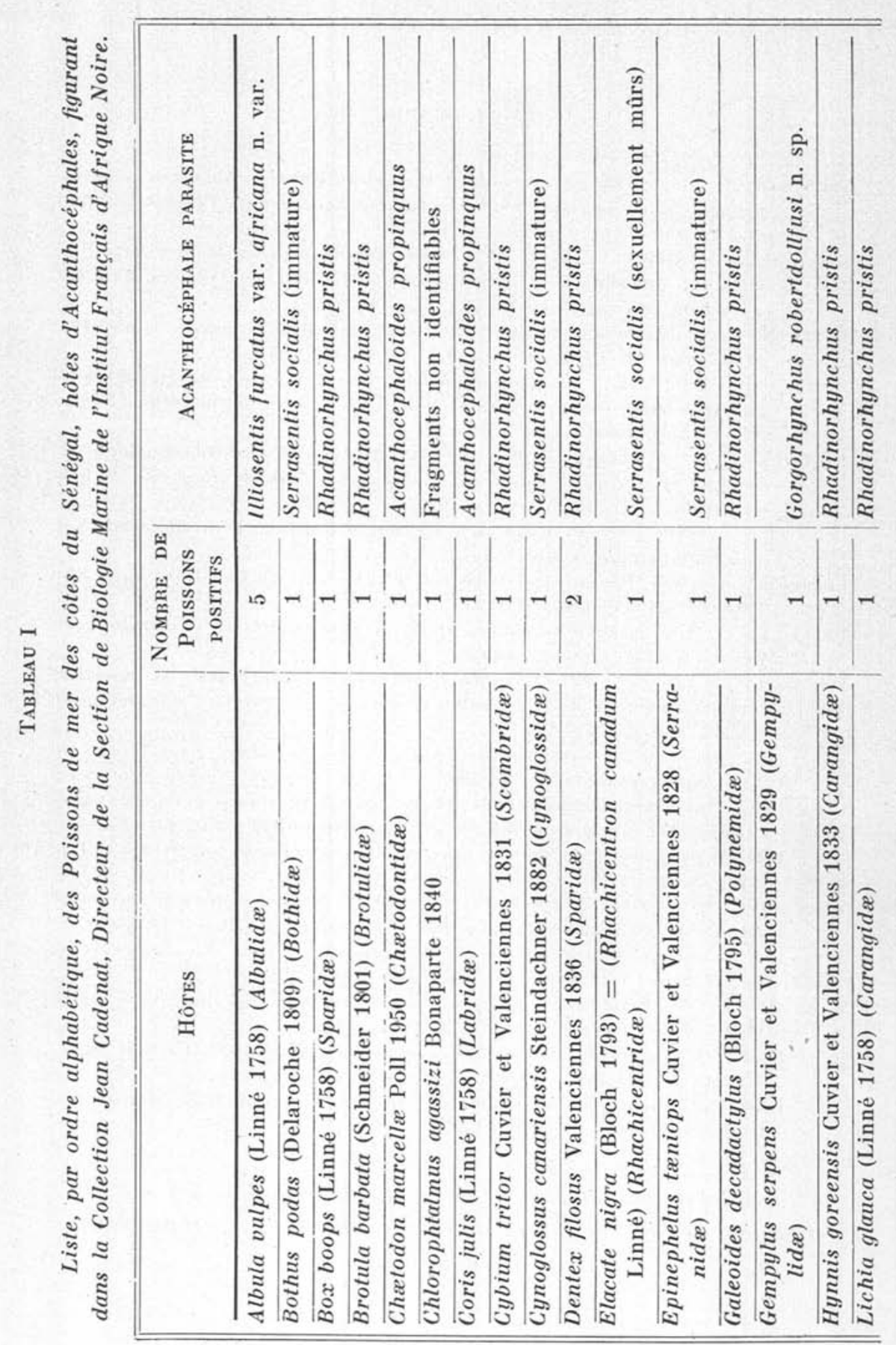




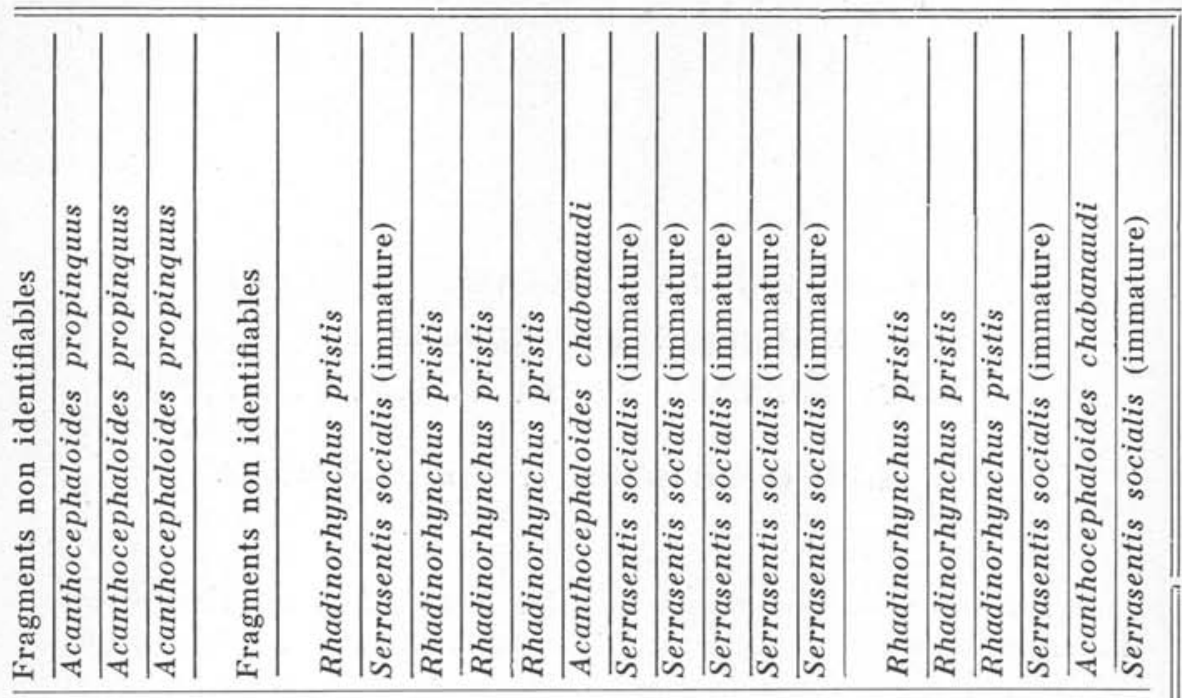

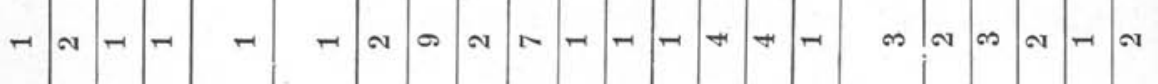

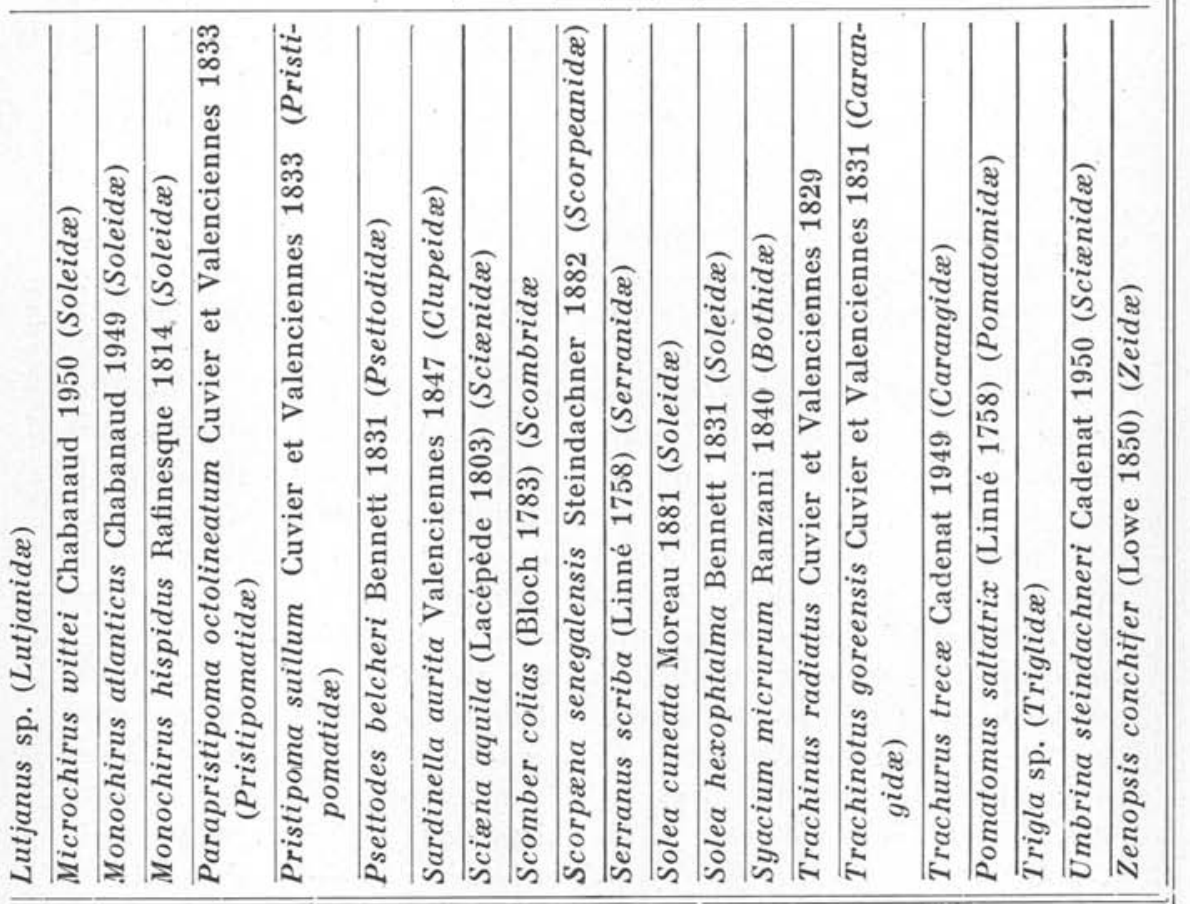

Ann. de Parasitologie, T. XXXI, $\mathrm{N}^{\circ} 3 .-1956$.

16. 